\title{
Optimal Robotic Path Planning Using Intelligent Search Algorithms
}

\author{
Baidaa AlKhlidi ${ }^{1}$, Ahmad T. Abdulsadda $^{2}$, Ali Al Bakri ${ }^{3}$ \\ 1,2,3 Communication Engineering Technical Department, Al Furat AlnAwast Technical University,Al Najaf, 54001, Iraq \\ E-mail: ${ }^{1}$ baidaa.alkhlidi@yahoo.com, ${ }^{2}$ coj.abdulsad@atu.edu.iq
}

\begin{abstract}
This investigate investigates the application of Adjusted Fuzyy Molecule Swarm Optimization (FPSO) to the versatile robot route issue in arrange to decide the briefest conceivable course with the least time required to travel from a beginning area to a goal area in a deterrent working zone. MPSO is being created in this ponder to progress the capability of customized calculations for a worldwide course. The proposed calculations decipher the environment outline spoken to by the framework show and develop an ideal or nearly ideal collision-free way. Reenactment tests appear the viability of the most recent organized calculation for portable robot course arranging. The programs are composed in MATLAB R2019a, and run on a $2.65 \mathrm{GHz}$ Intel Center i5 and 7 GB Smash computer. Changes proposed in MPSO and cuckoo look calculation fundamentally point to resolve the untimely merging issue related with the beginning PSO. A mistake calculate is demonstrated within the MPSO to guarantee meeting of the PSO. FPSO points to handle another issue which is the populace may incorporate a few infeasible ways; an updated strategy is tired the FPSO to fathom the issue of the infeasible street. The discoveries illustrate that this calculation has huge potential to fathom the course arranging with satisfactory comes about in terms of decreasing remove and time for execution.
\end{abstract}

Keywords- Modified Fuzzy Particle Swarm Optimization, Local Route Management, Intelligent Wireless Robot, Robot Navigation.

\section{INTRODUCTION}

The subject of the ideal robot way arranging was proposed around within the mid of the 1960's. Firstly, let characterized the Robot Way Arranging (RPP) as a most imperative issue within the route of moving versatile robots as of late, particularly when numerous versos and maladies spread out through the nation and some way or another mechanical technology gets to be more proficient way to control the human life things such as: driving the trains, body watch and stores receptionist etc. within the defilement region. The word "Robot" is initially determined from the word 'Czech' "Robota" which implies fundamentally a way for doing things physically. For the most part Oxford word reference was characterized the word "Robot" as a machine that's looking like a human being which have been utilizing to moving and control materials, otherwise it may well be characterized as the parts devices or specialized gadgets through the variable modified movement to be doing the execution of a assortment of errands [1-4].

Route may be a significant capacity for numerous robots that claim to be portable and moving remotely. It incorporates the capacity of the robot to be moving based upon its information or its controller activity and it is detecting data from the natural that where it is, so that it can reach its target as proficiently and before long as conceivable. Route includes numerous things, like mapping, detecting, acting arranging, equipment and control efficiencies. The point of portable robots is to travel from beginning point area to the ultimate point target area with the littlest remove and ideal way. In reality, there are numerous streets for the robot to reach its target, but the ideal way is chosen agreeing to a few ideal rule's calculations such as: the most limited remove, the slightest vitality devouring or the slightest time [5-8]. These rules are been seen as an optimization issue since its objective is to explore for a way with the most limited remove beneath certain constraints such as maintain a strategic distance from any collision by the deterrents that will happen in their moving in a certain environment [9-12].

The mapping assignment is considered to be one of the most parameters of talking about really independent mobile robot navigation. This is often the primary step is tending to within the issue of robot route. Way arranging is the clearest viewpoint of route that would be replied the taking after address: Which is the ideal and culminate way for the robot to move from half quart 1 to the point 2? Given a outline and the a begin point and the ultimate target point area, way arranging distinguishes a trajectory automatically that will cause the robot to discover the ultimate objective point area by tackling the vital ideal issue, as the robot have to be choose which way must be taken after to discover the goal point without making any collision with the impediments in the event that there are [13-17].

The most versatile robot planning to have three basic parts which are (appeared Fig. 1): the detecting square, controller piece, and engine drive piece. The detecting piece utilizing for giving the all-natural data from the exterior word. The controller piece, which creates the control flag activity based upon what the detecting piece is detected and at last the engine square, which is gotten the KMJWZ signal from the controller piece and these flag would be inferred the engines to move clockwise and counter clockwise with a particular revolution point and the amassing for the engines turn the robot would be moving and doing a particular movements [18-19].

In this paper, we proposition a fluffy particle swarm and worldwide cuckoo look calculation, the rest of the paper organized as taking after: area II presents the issue detailing with details of fluffy rules that has been utilized and rule of 
PSO and cuckoo look calculation. Segment III presents the proposed calculation setup and flowchart, segment IV presents the reenactment comes about, last the conclusion is commented in segment $\mathrm{V}$.

\section{PRoblem MATHEMATICAL FORMULATION}

\section{A. Fuzzy rule}

Let us recollect the kinematic reveal of a wheeled transportable robotic (WMR) and the situation of the robotic inside the aircraft as confirmed up in Fig. 1.

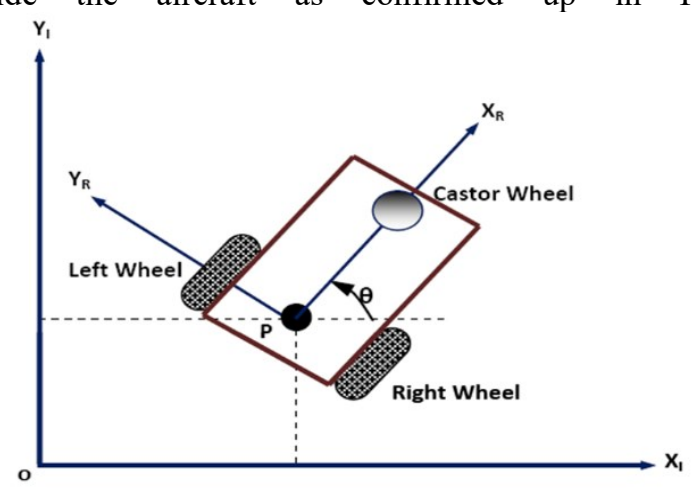

Fig. 1. Robotic modelling shape

For the show essential building of ANFIS, we acknowledge that the soft inducing system practical has two information sources $\mathrm{x} 1$ and $\mathrm{x} 2$ and one abdicate $\mathrm{y}$. Expect the feathery inducing system contains two IF-THEN rules of the Takagi-Sugeno sort [5] as showed up in Fig. 2.

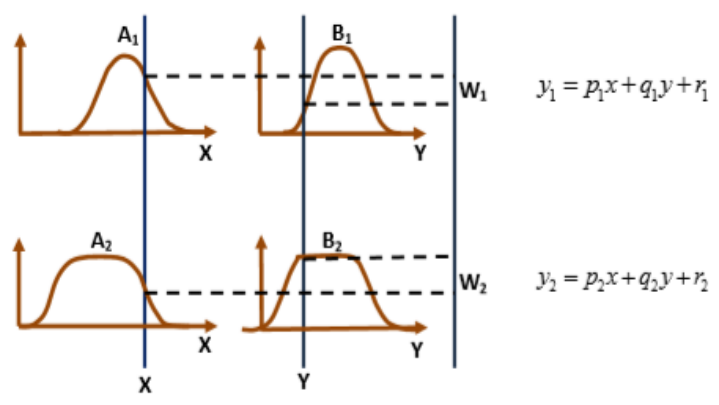

Fig. 2. Fuzzification membership functions.

It is obviously the fuzzy rules can be built as:

Rule 1: IF $\mathrm{x}_{1}$ is $\mathrm{A}_{1}$ AND $\mathrm{x}_{2}$ is $\mathrm{B} 1$ THEN

$$
\mathrm{y} 1=\mathrm{p}_{1} \mathrm{x}_{1}+\mathrm{q}_{1} \mathrm{x}_{2}+\mathrm{r}_{1}
$$

Rule 2: IF $\mathrm{x}_{1}$ is A2 AND $\mathrm{x} 2$ is B2 THEN

$$
\mathrm{y}_{2}=\mathrm{p}_{2} \mathrm{x}_{1}+\mathrm{q}_{2} \mathrm{x}_{2}+\mathrm{r}_{2}
$$

Where, A1, A2, B1, and B2 are the etymological delineation of information sources $\mathrm{x} 1$ and $\mathrm{x} 2$ independently. $\{\mathrm{p} 1, \mathrm{q} 1, \mathrm{r} 1\}$ and $\{\mathrm{p} 2, \mathrm{q} 2, \mathrm{r} 2\}$ are the following parameters, and $\mathrm{y} 1$ and $\mathrm{y} 2$ are the assessed abdicate of the framework. Now, considering the target of the proposed ANFIS navigational controller is to predict the coordinating point of the flexible robot. Here, we consider the flexible neuro-fluffy controller Fig. 3 beneath the thought of four data parameters, for illustration Front catch partition (FOD) (X1), Right prevention partition (Bar) (X2), Cleared out obstruction partition (LOD)(X3) and Heading point (HA)(X4). This information is assembled by an assortment of sensors which are mounted around the exterior of the robot.

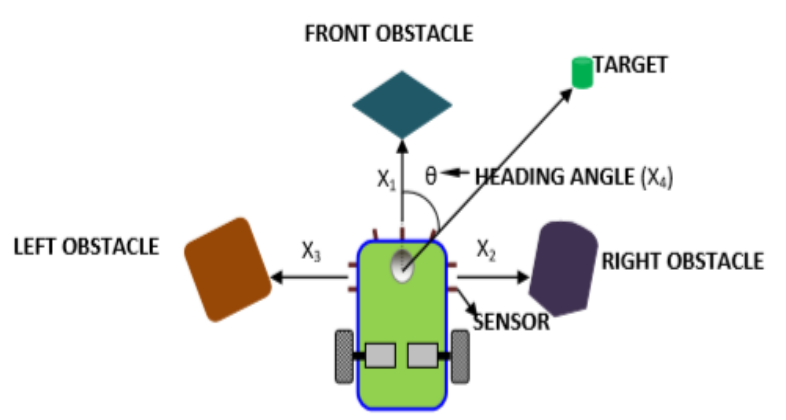

Fig. 3. Robotic fuzzification modelling [20]

Right presently molded kind of enrollment capacities are taken into consideration and every statistics variable has 3 chime assist capacities (MF) or semantic variables A1(Near), A2(Medium) and A3(Far), B1(Near), B2(Medium) and B3(Far), C1(Near), C2(Medium) and C3(Far), D1(Negative), D2(Zero) and D3 exclusively; at that factor a Takagi-Sugenokind soft derivation gadget IF-THEN benchmarks are constructed as takes after;

$\operatorname{IF}\left(x_{1}\right.$ is $A_{i}$ and $x_{2}$ is $B i$ and $x 3$ is $C i$ and $x 4$ is $\left.D i\right)$

THEN

(rotating angle

$$
\left.(\beta n)=p_{n} x_{1}+q n x 2+r_{n} \times 3+s n x 4+u_{n}\right)
$$

A, B, C and D are the fluffy enrollment units for the enter elements $\mathrm{x} 1, \mathrm{x} 2, \mathrm{x} 3$ and $\mathrm{x} 4$ separately. The Bell-molded enrollment evaluation of a soft set $\mathrm{S}$ ( $\mathrm{Ai}, \mathrm{Bi}, \mathrm{Ci}$ and $\mathrm{Di}$ ) and it makes a decision how tons the given reasserts of information (X1, X2, X3 and X4) satisfy the quantifier S. The hobby capacities for A, B, C and D are the chime form capacities and that is confirmed up in Fig. 4 [22-25].

$$
\mu_{A i}=\frac{1}{1+\left|\left(\frac{x_{i}-c_{i}}{a_{i}}\right)^{2}\right|}
$$

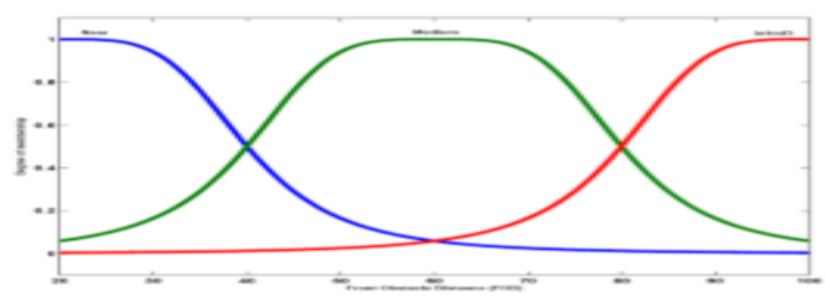

Fig. 4. Member ship input output function.

Variable $\mathrm{a}_{\mathrm{i}}$, and $\mathrm{c}_{\mathrm{i}}$ are parameters that manage the interior, width and incline of the Bell-molded potential of center 'I' independently. They are something else known as the antecedent or cause parameters. Changing those parameters will provide the diverse shapes of a chime molded potential as required as according to the educational list for the difficulty characterized. The soft interest potential may be picked in any structure, for case, triangular, Gaussian, trapezoidal, chime molded, and so forward. As a run the show, we choose the chime molded one with the foremost 
extraordinary worth proportional to at least one and slightest worth proportionate to.

\section{B. Modified Particle swarm optimization algorithm}

Basically, the MPSO, much the same as the PSO, comprises of a mass of particles that aggregately filter within the journey space for the around the world perfect. Upgrades are overwhelmingly endeavoring to address the issue of inopportune mixing related with the standard PSO. These enhancements ordinarily Endeavor to require care of this issue by growing the decent assortment of courses of action within the swarm. Within the MPSO a goof calculate is shown, to ensure that the PSO joins. MPSO Endeavor to address another issue which is the populace may consolidate various infeasible ways, which have terrible effect on the presentation of the calculation. Within the MPSO, the infeasible ways are not arranged of in any case can be changed to be doable way, Fig. 5 appears the schematic graph for the issue explanation.

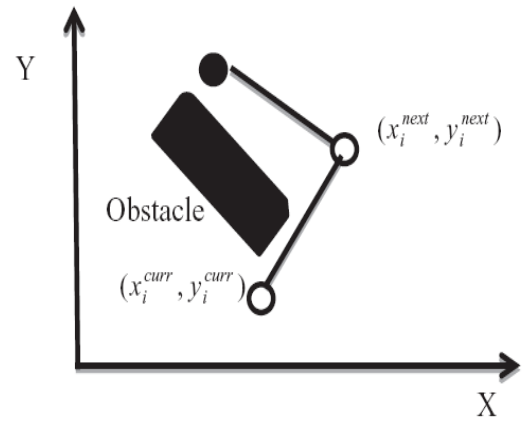

Fig. 5. PSO schmenatic diagram, [26].

Consider at first, the robotic $\mathrm{Ri}$ is about in the region at $\left(\mathrm{xi}^{\mathrm{curr}}, \mathrm{yi}^{\mathrm{curr}}\right)$. We want to find the following region of the robotic $\left(\mathrm{xi}^{{ }^{\text {next }}}, \mathrm{yi}^{\text {next }}\right)$ via way of means of joining of the 2 focuses between $\left\{\left(\mathrm{xi}^{\text {curr }}, \mathrm{yi}^{\text {curr }}\right),\left(\mathrm{xi}^{\text {next }}, \mathrm{yi}^{\text {next }}\right)\right\}$ and $\left\{\left(\mathrm{xi}^{\text {next }}\right.\right.$, $\left.\left.\mathrm{yi}^{\text {next }}\right) ;\left(\mathrm{xi}^{\text {goal }}, \mathrm{yi}^{\mathrm{goal}}\right)\right\}$ ought now no longer touch the deterrent on earth map, as spoke to in "Fig. 5", and bounds the all-out manner period from current scenario to an goal scenario without contacting the obstruction via way of means of framing limitation. At that factor the goal paintings F1, which comes to a decision the path manner period for $\mathrm{n}$, variety of robots, is

$$
F_{1}=\sum_{i=1}^{n}\left\{\sqrt{\left(\left(x_{i}^{\text {curr }}-x_{i}^{\text {next }}\right)^{2}+\left(y_{i}^{\text {curr }}-y_{i}^{\text {next }}\right)^{2}\right)}\right\}
$$

As PSO is impelled by the social thought of winged animal running, each juvenile can be considered as one particle. So, each atom has its exceptionally claim position and speed. The circumstance of particle which fulfill perfect wellness regard degree is picked as the around the world best position. Fig. 6 appears the structure of PSO.

The beginning situation for every molecule is the beginning stage of the way, at that point the principal position and speed of every molecule is created arbitrarily yet constrained to the limits of the inquiry space. The molecule's position put away in a network with size of $2 \times$ 1 , where 1 is the quantity of particles. The first and second line of the framework relate the $\mathrm{x}$ and $\mathrm{y}$-facilitate for all particles, individually. The directions of start and objective focuses are $x_{s}, y_{s}$ and $x_{g}, y_{g}$ individually, and middle of the road focuses in the way are spoken to by $x_{n}, y_{n}$.

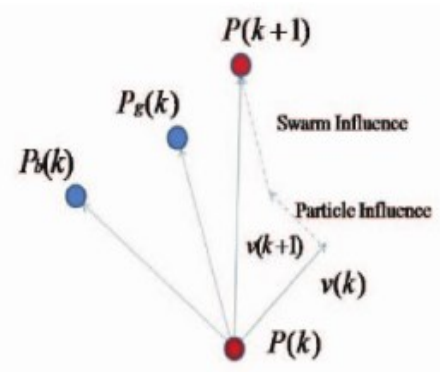

Fig. 6. MPSO robotic path planning [27].

The Fitness Function Evaluation, the molecule's ways are assessed during each progression. Assessment work is utilized to give a proportion of how particles have acted in the issue space. In the MPSO, two parameters (the separation among focuses and travel time) showed by the particles are utilized to ascertain the molecule wellness (p_fitness). Since the wellness should increment as the separation and time diminishes, the wellness work for every molecule of a doable way is ended up being a minimization issue and it is assessed as:

$$
\text { Fitness }=\frac{1}{w_{d} d\left(p_{\left.i \cdot p_{i+1}\right)}\right.}+w_{t} t\left(p_{i} \cdot p_{i+1}\right)
$$

where $\mathrm{w}_{\mathrm{d}}$ and $\mathrm{w}_{\mathrm{t}}$ are weighting factor for separation and travel time separately, $\mathrm{d}\left(\mathrm{p}_{\mathrm{i}}, \mathrm{p}_{\mathrm{i}+1}\right)$ means Euclidean separation between direct $\mathrm{Pi}$ toward the following point $\mathrm{p}_{\mathrm{i}+1}$ for a similar molecule and $t\left(p_{i}, p_{i+1}\right)$ indicates time taken by the molecule to cover from $\mathrm{pi}$ to $\mathrm{p}_{\mathrm{i}+1}$ :

$$
d\left(p_{i} \cdot p_{i+1}\right)=\sqrt{\left(x_{i}-x_{i+1}\right)^{2}-\left(y_{i}-y_{i+1}\right)^{2}}
$$

And

$$
t\left(p_{i} \cdot p_{i+1}\right)=\frac{d\left(p_{i} \cdot p_{i+1}\right)}{v_{i}}
$$

where $x_{i}$ and $y_{i}$ are molecule's present even and vertical positions, $x_{i+1}$ and $y_{i+1}$ are molecule's next flat and vertical positions, and $v_{i}$ signifies the speed of the molecule when going from $p_{i}$ to $p_{i+1}$. The MPSO calculation is instated with various particles and afterward scans for ideal. The situation of a molecule is impacted by the best position visited without anyone else which is alluded to as molecule best "pbest", and the best situation in the entire swarm which is alluded to as a worldwide best "gbest". A molecule refreshes its position and speed utilizing the accompanying conditions [17]:

$$
v x_{i+1}=w_{1} v x_{i}+c_{1} r_{1}\left(p_{\text {best }}-x_{i}\right)+c_{2} r_{2}\left(g_{\text {best }}-x_{i}\right)
$$

$$
\begin{aligned}
& \text { And } \\
& \qquad v y_{i+1}=w_{1} v y_{i}+c_{1} r_{1}\left(p_{\text {best }}-y_{i}\right)+c_{2} r_{2}\left(g_{\text {best }}-y_{i}\right)
\end{aligned}
$$

Where the subjective fragment, (pbest-xi), talks to the molecule's claim understanding with regard to where the most excellent course of action is and the social segment, 
(pbest-yi) talks to the conviction of the entire swarm approximately where the leading course of action is. vxi +1 and vyi +1 is the revived molecule's speed. vxi +1 , vyi +1 and xi, yi are current molecule's speed and position along $\mathrm{x}$ and $\mathrm{y}$-heading independently. Reviving speeds is the way that enables the particle to see around its person best position and around the world best position. Fearlessness calculate, c1 and swarm-certainty calculate, c2 causes particles to have the capacity of self-rundown and figure out how to the finest of the swarm, and draw close to the finest circumstance of its claim fair as interior the swarm. The dormancy weight (w) was to begin with displayed by [18]. This term fills in as a memory of past speeds and it utilized to control the impact of the past speed on the show speed. The estimation of inertness calculate (w) is within the scope of $[0,1] . \mathrm{r} 1$ and $\mathrm{r} 2$ are sporadic numbers interior the intervals of $[0,1]$. These parameters straightforwardly influence execution of PSO.

In the fundamental idea of PSO calculation no blunder model is utilized for refreshing the position. In any case, in the MPSO these blunders are displayed to guarantee that the PSO merges, the altered position update condition for every molecule with the mistake is resolved as follow:

$$
x_{i+1}=x_{i}+v x_{i+1}+r * \text { Errorx }
$$

And

$$
y_{i+1}=y_{i}+v y_{i+1}+r * \text { Errory }
$$

where $\mathrm{x}_{\mathrm{i}+1}$ and $\mathrm{y}_{\mathrm{i}+1}$ are the new locations along $\mathrm{x}$ and $\mathrm{y}$-axis, respectively, $r$ is an arbitrary number, enormous estimations of this parameter prompt worldwide inquiry, while little qualities prompts fine, neighborhood search, which is appropriate when calculation meets. Errorx and Errory are position blunder along $\mathrm{x}$ and $\mathrm{y}$-arrange for all particles, individually, and it's between current molecule position and the objective position. These blunders are accustomed to altering the molecule's course to make a beeline for the objective.

$$
\begin{aligned}
& \operatorname{Errorx}_{i}=x_{p}-x_{t} \\
& \operatorname{Errory}_{i}=y_{p}-y_{t}
\end{aligned}
$$

The error value is resolved for every particle where $x_{p}$ and $\mathrm{y}_{\mathrm{p}}$ are the present directions of the individual particle and $\mathrm{x}_{\mathrm{t}}$ and $\mathrm{y}_{\mathrm{t}}$ are the objective directions. In light of the above conditions, the following conceivable speed and position of every molecule is resolved. On the off chance that the following conceivable position dwells inside the hindrance space, the impediment evasion some portion of the calculation is utilized and the Distinguish Algorithm (DA) is utilized to check the ways.

\section{Cuckoo Search optimization Algorithm}

Let the robot is at begin point (An) and moves to the objective point (B) in nature as showed up in Fig. 7. The line $A$ to $B$ is the perfect course for the robot to reach at the objective with the perfect way length. The robot has to arrive at the objective while keeping up a vital remove from obstacles show within the soil. At the point when the robot comes to approach the obstructions, its sensors recognizes obstacles. At that point the CS calculation will be ordered to preserve a key separate from the preventions show within the perfect objective way. Note that at anything point an obstruction falls interior the periphery of the robot identifying extend, the CS calculation will be begun to find the finest domestic circumstance for the robot (as per target work worth) to stay away from impacts with obstacles present within the objective way. Each arrangement within the issue space is related with a numeric worth. Within the CS calculation, a retirement finance of the most excellent quality (plausibility of making cuckoo chick) will prompt another age. In this way, the nature of a cuckoo's egg (modern cuckoo) is distinguished with the progressed way length of the robot. As clarified within the over meta-heuristic improvement issue, each movement to be moved by the robot is decided subordinate on the division between the places of the homes (cuckoo) to the objective and the obstacles show within the cloud condition [28-30].

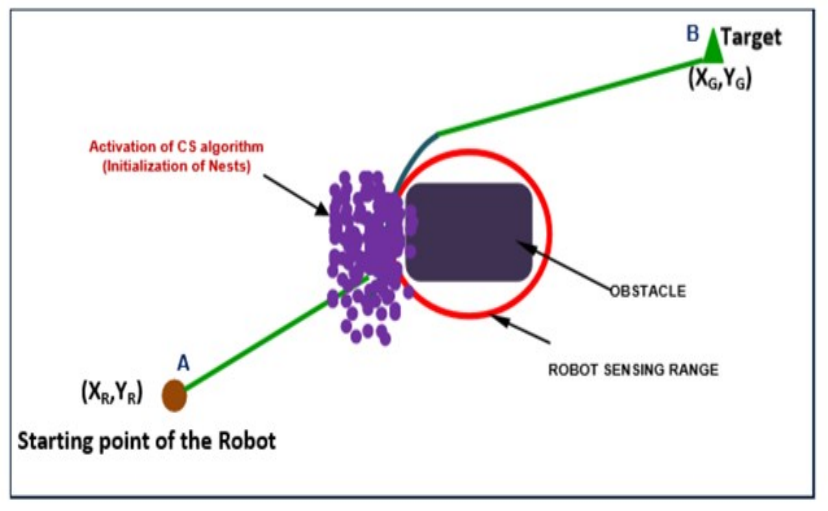

Fig. 7. CS robotic path planning.

A-When all is said in done, the robot way organizing for the foremost portion depends on the going with two hones, an) Impediment avoiding conduct the hindrance avoidance conduct is utilized to go without from hitting with preventions, (for example, dividers) display within the earth. The situation of the finest domestic (cuckoo) have to be have kept up the foremost extraordinary secure great ways from the closest hindrance. The Euclidean division between the finest domestic and closest catch is decided by the going with condition as distant as target work as takes after:

$$
D_{N-o b s}=\sqrt{\left(x_{o b}-x_{i}\right)^{2}+\left(y_{o b}-y_{i}\right)^{2}}
$$

where, $\mathrm{x}_{\mathrm{i}}$ and $\mathrm{y}_{\mathrm{i}}$ are the nest (cuckoo) position at $\mathrm{x}$ and $\mathrm{y}$ arranges. (DN-OB) is the Euclidean good ways from the home (cuckoo) to the closest hindrance. The closest snag to the robot can be determined by the accompanying articulation:

$$
D_{R-o b s}=\sqrt{\left(x_{o b}-x_{R}\right)^{2}+\left(y_{o b}-y_{R}\right)^{2}}
$$

B- Target searching for conduct in its most clear structure, the move to target conduct can communicate the yearning to move to a specific robot position. The situation of the leading domestic (cuckoo) have to be have kept up the base great ways from the objective [15]. The Euclidean division 
between the domestic and objective is decided by the going with condition with respect to target work as takes after:

$$
D_{N-\text { target }}=\sqrt{\left(x_{t}-x_{i}\right)^{2}+\left(y_{t}-y_{i}\right)^{2}}
$$

where, $\mathrm{xt}$ and $\mathrm{yt}$ are the objective location at $\mathrm{x}$ and $\mathrm{y}$ organizes. (D N-target) is the base Euclidean good ways from the home (cuckoo) position to the robot. In light of the over two significant practices of the robot, the target capacity or the fitness function of each nest for the way streamlining issue can be communicated as follows:

$$
F(\text { fitness })=C_{1} \underbrace{\frac{1}{\min \left\|D_{N-o b s}\right\|}}_{N-\text { obs }}+C_{2}\left\|D_{N-\text { traget }}\right\|
$$

\section{PROPOSED ALGORITHM SETUP}

Generally, in this section presents the flowchart and algorithms step for both MPSO and CS algorithms. Firstly Fig. 8 shows the main steps flowchart for the MPSO.

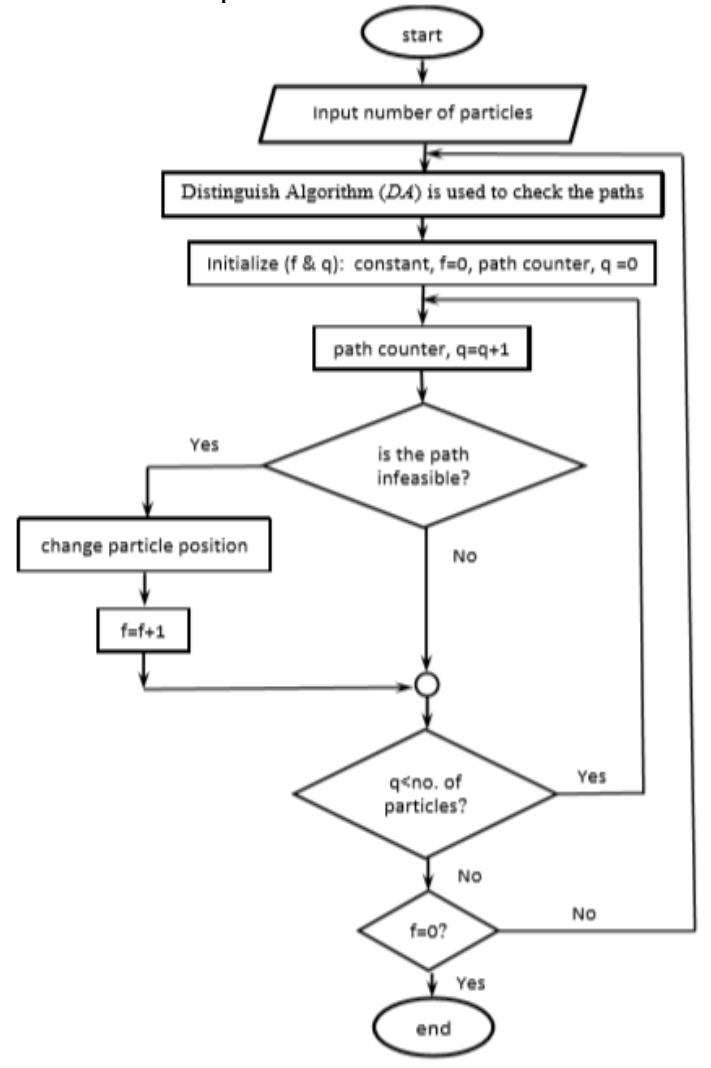

Fig. 8. MPSO flowchart.

Now, we present the flowchart of the CS shown in Fig. 9. where the main steps are:

Objective Function $\mathrm{g}(\mathrm{k})$, where $\mathrm{k}=(\mathrm{k} 1, \mathrm{k} 2, \ldots \ldots \mathrm{kd}) \mathrm{T}$ Let the starting population of the host nest ' $n$ ' $\mathrm{ki}(\mathrm{i}=1,2 \ldots \ldots \mathrm{n})$ While ( $\mathrm{t}<$ maximum generation) or (Criteria stop)

Randomly get a cuckoo by the levy flight

If $(\mathrm{Fi}>\mathrm{Fj})$ then Then $\mathrm{j}$ is replaced by the new solution End In the place of the abandoned worst nest (pa) new nest are built

Keep the best solutions (or quality solutions with the nest) Solution will be numbered to find the current best solution End while Process
The result end in case of no detection of an obstacle it will certainly travel towards its destination after that no intelligent computation is required for the detection of the destination.

The main purpose for using these two important algorithms because it still working in complex environments and in multiple robots' case.

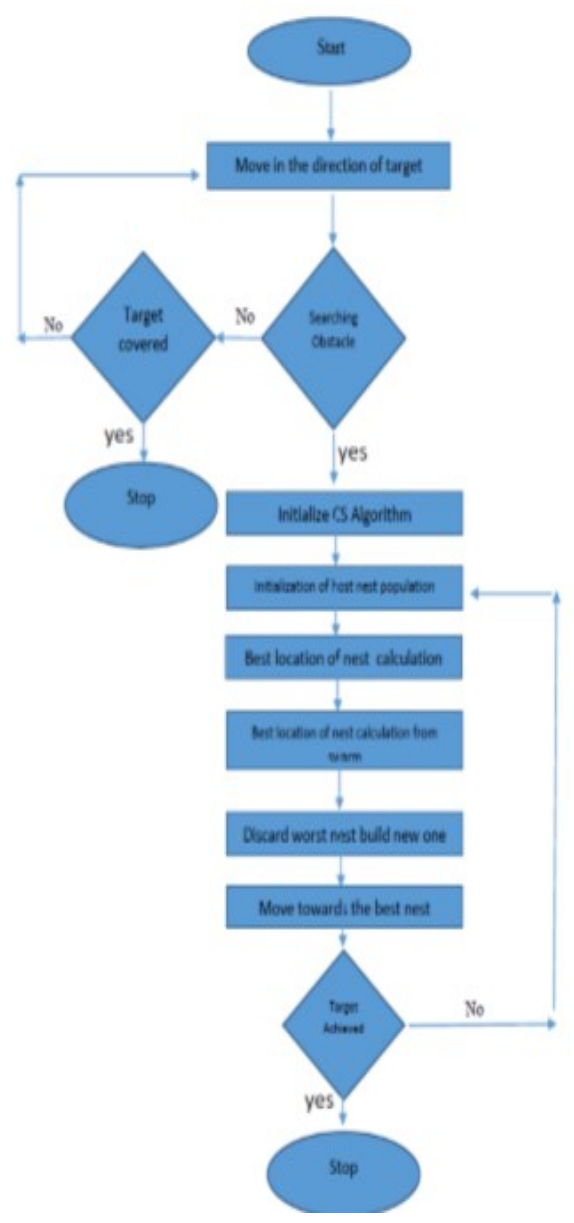

Fig. 9. Cuckoo search algorithm.

\section{SIMULATION RESULTS}

\section{A. Fuzzy Rule Results}

The first algorithm which is the traditional algorithm studied here to show how performance it gives when it faces the obstacles. Table II illustrated the simulation setup that been used in this algorithm.

Table I. Fuzzy simulation parameters set up.

\begin{tabular}{|c|c|c|}
\hline No. & Description of the parameters & Simulation setup value \\
\hline $1-$ & Maps size & $500 \times 500$ area units \\
\hline $2-$ & Initial point & {$[50,50]$} \\
\hline $3-$ & Final point, goal point & {$[450,450]$} \\
\hline $4-$ & speed & 10 arbitrary unit \\
\hline $5-$ & Max speed & 10 arbitrary unit \\
\hline $6-$ & Safety distance & 10 length unit \\
\hline $7-$ & Threshold distance & 30 length unit \\
\hline $8-$ & Acceleration & 10 \\
\hline
\end{tabular}


The main drawback point for the potential field algorithm is that when we have used multiple irregular obstacles it did not recognize the target point and the collision happened. So that, the simple environments which are consider simple number of barrier and obstacles the robot could be using the potential algorithm as efficient algorithm to find the target point but essentially this algorithm would be broken up whenever the number of obstacles increase especially if it were irregular obstacles. Now, let exam the adaptive fuzzy algorithm with irregular complex environment as shown in Fig. 10.

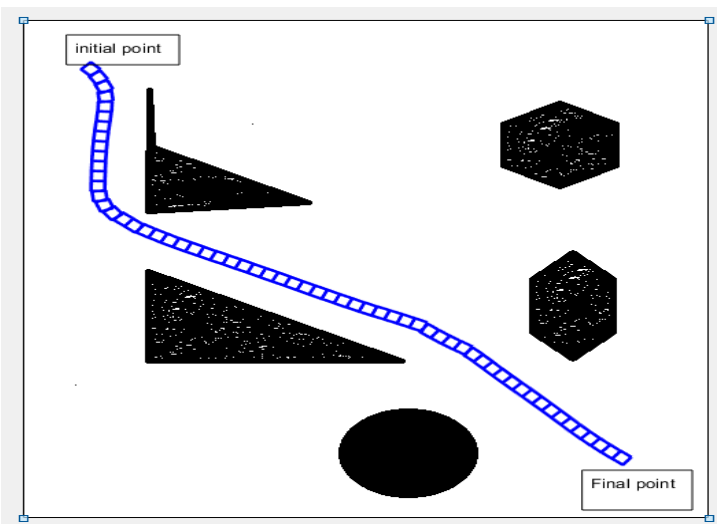

Fig. 10. Robotic planning simulation result using fuzzy rule.

\section{B. Modifier Particle swarm Optimization Results}

Once of the most important drawback in the adaptive fuzzy algorithm in the robotic path planning it takes too longer time and it needs expertise to build up the fuzzification rules lookup table. One main proposal to solve this problem and find algorithm to update their status automatically without needing to expert's information in prior. First let defined the main parameters that the PSO algorithm used to find the target point, Table II listed the all parameters and its description.

Table II. MPSO simulation setup parameters.

\begin{tabular}{|c|c|c|}
\hline No. & Parameters description & value \\
\hline $1-$ & Number of particles & 50 \\
\hline $2-$ & $\mathrm{C}_{1}$ (personal learning Coefficient) & 1.5 \\
\hline $3-$ & $\mathrm{C}_{2}$ (global learning coefficient) & 1.5 \\
\hline $4-$ & $\mathrm{W}_{1}$ (Inertia Weight) & 1 \\
\hline $5-$ & $\mathrm{W}_{2}$ (Inertia Weight Damping Ratio) & 0.98 \\
\hline $6-$ & Swarm size & 50 \\
\hline
\end{tabular}

We create an environment of $6 \times 6$ units, the unit is assumed to be meter. The initial point and final point for the robot are respectively represented by the yellow square and the green star, and the robot is considered as a point, with consideration the guard space that is explained extensively in chapter three. The obstacles are uniformly placed throughout the area. All obstacles are regular shape with the circle and square. Fig. 11 shows how the robots moving from the initial point to the final point rapidly and avoid the collision with obstacles, Fig.
12 shows the best global fitness function which is represented the shortest distance and smallest reaching time.

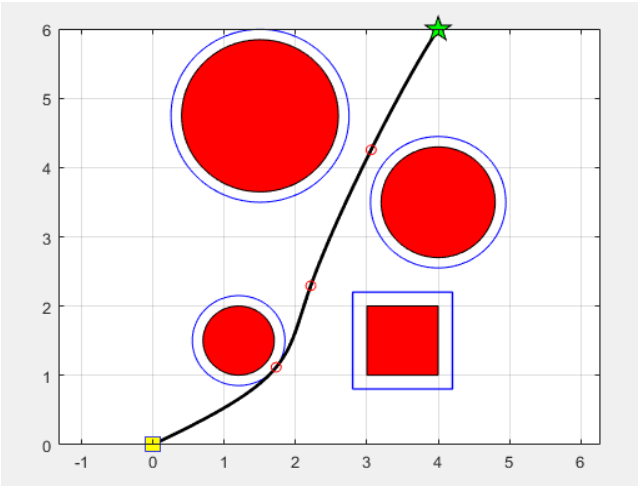

Fig. 10. MPSO simulation result one robot.

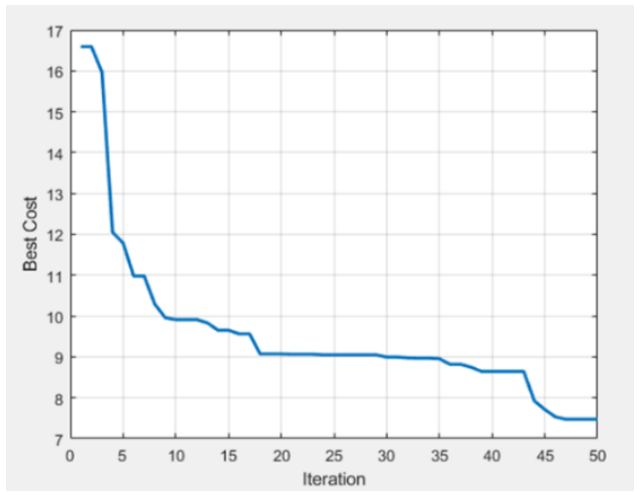

Fig. 11. Global fitness function.

To investigate the impact of different swarm sizes on MPSO efficiency, various numbers of particles (i.e., 5, 10, 50 , and 100) have been taken into account. The particle size was chosen on the basis of the trial and error, since the literature did not suggest swarm size. Different combinations of parameters were checked in the PSO and the best results obtained are: weighing factor values in fitness function $\beta=100$ and $w_{1}=1$, weighing factor inertia $w=0.98$. At the start of optimization, the swarms with low initial inertia weight converged fairly quickly. The cognitive scaling values chosen and the social scaling variables are $\mathrm{c} 1=\mathrm{c} 2=1.5$. The best observed simulation results for the environment are shown in Fig. 12 with different particle amounts (swarm size).

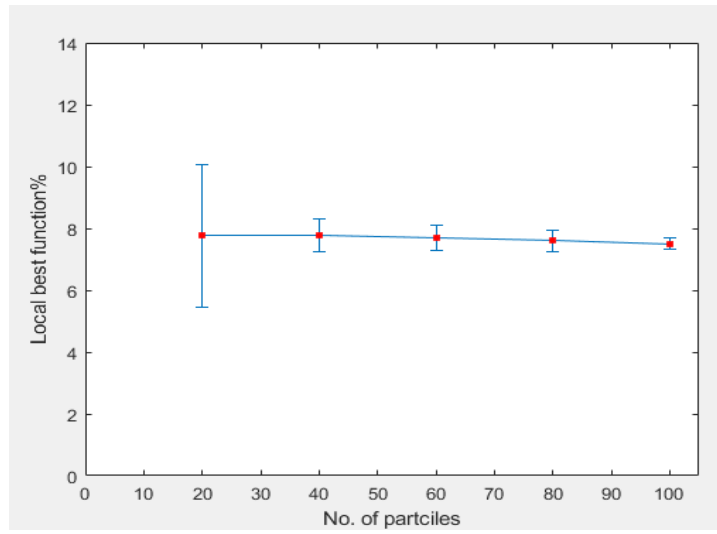

Fig. 12. Varices change when the number of particle change. 


\section{Cuckoo Search Results}

CS is global optimization technique it is using if the map is becoming complex. The simulation results are shown on Fig. 13.

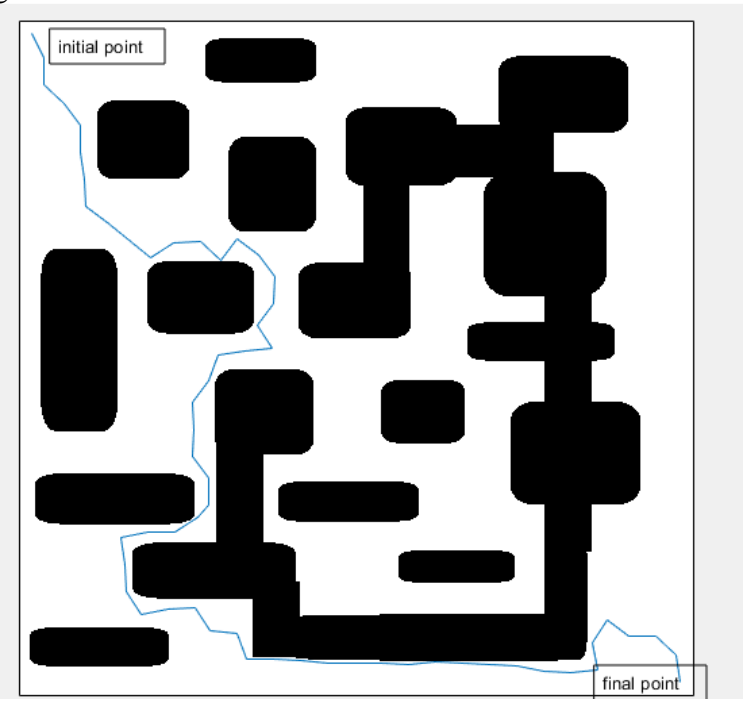

Fig. 13. Simulation results of one robot using CS.

\section{CONCLUSION}

This paper provided the novel approach for evaluating parameters involved in multi-robot route planning strategies with the aid of the nature-inspired algorithm, which involves Particle Swarm optimization and Cuckoo Search, via the sequence of simulation maps, graphs and tables, the following algorithm has been properly demonstrated. It was found by seeing the graphs guided by the necessary parameters that the two algorithms PSO and CS obey the different traverse mechanisms in the complex world. On the one hand, in the case of Cuckoo search, all the robots start from the same point and arrive at the same point after crossing the world, while on the other hand the agents obey the PSO optimization strategy Unable to get going and finish at the same point. That suggests that the strategy of maximizing the cuckoo hunt is far more feasible and successful in contrast with the PSO. Our future research will concentrate on the study of parameters on the dual existence of both the algorithm CS and PSO. We will merge both the PSO and CS algorithms together to create a hybrid algorithm for future research Comparing the results with PSO and CS independently and evaluating the parameters based on robots traversing, we will approximate the performance of the algorithm and its suitability to the route planning technique.

\section{REFERENCES}

[1] Chatila, R., Lacroix, S., Simeon, T., Herrb, M.: Planetary exploration by a mobile robot: mission teleprogramming and autonomous navigation. Auton. Robots 2(4), 333-344 (1995)

[2] Singer, P.: Military robotics and ethics: a world of killer apps. Nature 477(7365), 399-401 (2011).

[3] Dario, P., Guglielmelli, E., Allotta, B., Carrozza, M.C.: Robotics for medical applications. IEEE Robot. Autom. Mag. 3(3), 44-56 (1996)

[4] Ahmadi, M., Stone, P.: A multi-robot system for continuous area sweeping tasks. In: IEEE International Conference on Robotics and Automation, pp. 1724-1729. ICRA (2006)
[5] Barraquand, J., Bruno, L., Latombe, J.-C.: Numerical potential field techniques for robot path planning. IEEE Trans. Syst. Man Cybern. 22(2), 224-241 (1992)

[6] Willms, A.R., Yang, S.X.: An efficient dynamic system for real-time robot-path planning. IEEE Trans. Syst. Man Cybern. Part B: Cybern. 36(4), 755-766 (2006)

[7] Bennewitz, M., Burgard, W., Thrun, S.: Finding and optimizing solvable priority schemes for decoupled path planning techniques for teams of mobile robots. Robot. Auton. Syst. 41 (2), 89-99 (2002)

[8] Gong, D.W., Zhang, J.H., Zhang, Y.: Multi-objective particle swarm optimization for robot path planning in environment with danger sources. J. Comput. 6(8), 1554-1561 (2011)

[9] Hao, W., Qin, S.: Multi-objective path planning for space exploration robot based on chaos immune particle swarm optimization algorithm. In: Deng, H., Miao, D., Lei, J., Wang, F.L. (eds.) AICI 2011, Part II. LNCS, vol. 7003, pp. 42-52. Springer, Heidelberg (2011)

[10] Rakshit, P., Banerjee, D., Konar, A., Janarthanan, R.: An adaptive memetic algorithm for multi-robot path-planning. In: Panigrahi, B.K., Das, S., Suganthan, P.N., Nanda, P.K. (eds.) SEMCCO 2012. LNCS, vol. 7677, pp. 248-258. Springer, Heidelberg (2012)684 S. Sharma et al.

[11] Ashraf, S., Budabbus, H., S.M.: Finding an optimal path planning for multiple robots using genetic algorithm. In: The 13th International Arab Conference on Information Technology, ACIT (2012.

[12] X. Li, F. Yu, and Y. Wang, "PSO algorithm based online self-tuning of PID controller," in 2007 International Conference on Computational Intelligence and Security (CIS 2007), 2007, pp. 128132.

[13] K. Ogata and Y. Yang, Modern control engineering, vol. 5. Prentice hall Upper Saddle River, NJ, 2010.

[14] A. Cervin, D. Henriksson, and M. Ohlin, "TrueTime 2.0 beta 5Reference Manual, Department of Automatic Control, Lund University, Sweden, June 2010. cit: 10.10. 2011.”.

[15] Y. Wanga and L. Heb, "Analysis and Simulation of Networked Control Systems Delay Characteristics Based on Truetime," Comput. Model. New Technol., vol. 17, no. 4, pp. 210-216, 2013.

[16] C.-C. Song, C.-F. Feng, C.-H. Wang, and D.-C. Liaw, "Simulation and experimental analysis of a ZigBee sensor network with fault detection and reconfiguration mechanism," in 2011 8th Asian Control Conference (ASCC), 2011, pp. 659-664.

[17] S. Hwang and D. Yu, "Remote monitoring and controlling system based on ZigBee networks," Int. J. Softw. Eng. Its Appl., vol. 6, no. 3, pp. 35-42, 2012.

[18] Á. Cuenca, J. Salt, A. Sala, and R. Pizá, “A delay-dependent dual-rate PID controller over an ethernet network," IEEE Trans. Ind. Informatics, vol. 7, no. 1, pp. 18-29, 2010.

[19] Peng, Chen, Dong Yue, and Min-Rui Fei. "A higher energy-efficient sampling scheme for networked control systems over IEEE 802.15. 4 wireless networks." IEEE Transactions on Industrial Informatics 12.5 (2015): 1766-1774

[20] Dolsma, J.: Nonlinear controller design based on genetic programming. Master's thesis, Technische Universiteit Eindhoven, Department Mechanical Engineering, Dynamics and Control Group, Eindhoven, 2007.

[21] Floreano, D., Husbands, P., Nolfi, S.: Evolutionary Robotics, pp.1423-1451. Springer, Berlin, 2008.

[22] Hoy, M., Matveev, A.S., Savkin, A.V.: Algorithms for collision-free navigation of mobile robots in complex cluttered environments: a survey. Robotica 33(3), 463-497, 2015.

[23] Kamel, M.E., Beji, L., Abichou, A., Mammar, S.: A novel obstacle avoidance approach for mobile robot system including target capturing. AIP Conf. Proc. 1107, 249-253, 2009.

[24] Minguez, J., Lamiraux, F., Laumond, J.: Motion Planning and Obstacle Avoidance, pp.827-852. Springer, Berlin , 2008.

[25] Mohanty, P.K., Parhi, D.R.: Optimal path planning for a mobile robot using cuckoo search algorithm. J. Exp. Theor. Artif. Intell. 28(1-2), 2016, 35-52.

[26] Prases K. Mohanty, Dayal R. Parith," Optimal path planning for a mobile robot using cuckoo search algorithm", Journal of experimental and theoretical artificial intelligence, 2014. 
[27] Nelson, A.L., Barlow, G.J., Doitsidis, L.: Fitness functions in evolutionary robotics: a survey and analysis. Robot. Auton. Syst. 57(4), 345-370, 2009.

[28] Oftadeh, R., Aref, M., Ghabcheloo, R., Mattila, J.: Bounded-velocity motion control of four wheel steered mobile robots. In: 2013 IEEE/ASME International Conference on Advanced Intelligent Mechatronics, 2013, 255-260.
[29] Štimac, G., Braut, S., žigulić, R.: Comparative analysis of PSO algorithms for PID controller tuning. Chin. J. Mech. Eng. 27(5), 2014, 928-936.

[30] Dierks, T., Jagannathan, S.: Control of nonholonomic mobile robot formations using neural networks. In: 2007 IEEE 22nd International Symposium on Intelligent Control, 2007, 132-137. 